\title{
Synaptic Gating and ADHD: A Biological Theory of Comorbidity of ADHD and Anxiety
}

\author{
Florence Levy*,I \\ 'School of Psychiatry, University of New South Wales, Prince of Wales Hospital, Randwick, Australia
}

\begin{abstract}
To derive a biologically based theory of comorbidity in Attention Deficit Hyperactivity Disorder (ADHD). Theoretical concepts and empirical studies were reviewed to determine whether the behavioral inhibition concept provided an understanding of biological processes involved in comorbidity in ADHD. Empirical studies of ADHD have shown comorbidity of ADHD and anxiety, while studies of behavioral inhibition tend to suggest independent disruptive and anxiety traits. This paradox can be resolved by an understanding of the dynamics of mesolimbic dopamine (DA) systems, where reward and delay of reinforcement are determined by tonic/phasic DA relationships, resulting in impulsive 'fearless' responses when impaired. On the other hand, comorbid anxiety is related to impaired synaptic processes, which selectively gate fear (or aggressive) responses from the amygdala at the accumbens. Monosynaptic convergence between prefrontal, hippocampal, and amygdala projection neurons at the accumbens allows the operation of a synaptic gating mechanism between prefrontal cortex (PFC), hippocampus, and amygdala. Impairment of this mechanism by lowered PFC inhibition allows greater amygdala input, and anxiety-related processes more impact, over the accumbens. In conclusion, a dual theory incorporating long-term tonic/phasic mesolimbic DA relationships and secondly impairment of PFC and hippocampal inputs to synaptic gating of anxiety at the accumbens has implications for comorbidity in ADHD, as well as for possible pharmacological interventions, utilizing either stimulant or axiolytic interventions. The use of DA partial agonists may also be of interest.

Neuropsychopharmacology (2004) 29, I589-1596, advance online publication, 28 April 2004; doi: I0. I038/sj.npp. 1300469
\end{abstract}

Keywords: ADHD; dopamine; comorbidity; synaptic gating; behavioral inhibition

\section{INTRODUCTION}

While there has been considerable interest in comorbidity in Attention Deficit Hyperactivity Disorder (ADHD), as an indicator of possible genetic subtypes (Biederman et al, 1992; Hudziac and Todd, 1993; Pliszka, 2000; Thapar et al, 2001), the biological basis of ADHD subtypes and comorbidity is still poorly understood. During the 1990s, inhibitory theories of ADHD have been largely dominated by Barkley's (1997a) theory of executive function, based on incapacity to inhibit prepotent responses, stopping ongoing responses, and inability to delay competing responses. Barkley (1997b) linked inhibition to four executive neuropsychological functions, which depended on it for effective execution: nonverbal working memory; internalization of speech (verbal working memory): self-regulation of affect-motivation-arousal; and reconstitution (behavioral analysis and synthesis). By linking his theory to neuropsy-

*Correspondence: F Levy, School of Psychiatry, University of New South Wales, Prince of Wales Hospital, Randwick NSW 2031, Australia, Tel: +61 2 93828213, Fax: +61 293828105 ,

E-mail: f.levy@unsw.edu.au

Received 13 January 2004; revised 18 March 2004; accepted 23 March 2004

Online publication: 26 March 2004 at http://www.acnp.org/citations/ Npp033604040 I5/default.pdf chological measures of executive function, Barkley implied that inhibition was predominantly a cortical function.

In 1983, at the third annual High Point Hospital on Attention Deficit Disorder, Herbert Quay (Quay, 1988a, b; 1993; 1997) suggested that Attention Deficit Disorder with Hyperactivity Disorder (ADDH) might be due to an underactive behavioral inhibitory system (BIS), as described by Gray (1987). Gray described two antagonistic systems exerting an influence on the possibility of a behavioral response. The behavioral activation system (BAS) responds to cues or conditional stimuli for reward and actively avoids punishment. The BIS inhibits behavior in response to cues or conditioned stimuli, which produce fear or anxiety, and the nonoccurrence of an expected reward.

While Quay's modification of Gray's theory had implications for a theory of comorbidity, there remain a number of clinical features of ADHD either not explained or not considered by either Barkley's executive functioning or Quay's BIS theories. Neither theory provides an explanation of the developmental features of ADHD, which often become apparent around age 3-4 years at a time when non-ADHD children are gaining more control over their behavior. The BIS theory postulates a lack of anxiety in psychopathy, but is unclear whether this also applies to ADHD. The paradox that children with ADHD often display 
fearlessness and impulsivity and yet may also manifest anxiety (Biederman et al, 1993; Graetz et al, 2001) has not been explained. The rapid and sometimes dramatic action of stimulant medications in remedying many of the symptoms of ADHD has also not been explained, nor have side effects of high medication doses such as tearfulness or obsessive rigidity been understood in biological terms. Seeman and Madras (1999) have reviewed mechanisms of action of antihyperactivity medications (methylphenidate (MPH) and dexamphetamine). They proposed that clinically relevant doses of stimulants might increase extracellular background levels of dopamine (DA) above that of action potential-dependent release of DA. As tonic DA is considered to mitigate the amplitude of phasic, action potential-dependent DA release, this results in relatively reduced amplitude of impulse-associated DA, less activation of postsynaptic DA receptors, and thus less psychomotor activity.

Finally, comorbidity and/or subtypes have not been addressed by either theory.

\section{Behavioral Inhibition}

The concept of inhibition has been widely applied, but remains somewhat mysterious and ubiquitous. While Barkley (1997a) applied the concept to executive functions, Gray (1987) applied the concept of inhibition to the BIS system (based on septo-hippocampal impairment), considered to be central in the control of anxiety. Quay (1997) suggested that the inability of children with ADHD to inhibit behavior in response to cues signalling fear was a core deficit. Oosterlaan (2001) suggested that an overactive BIS was the underlying substrate for temperamental inhibition and might predispose a child to the development of anxiety disorders. Thus, the BIS theory postulates opposing states for anxiety and ADHD (Oosterlaan and Sergeant, 1996). Barkley's concept of inhibition in ADHD is predominantly a cortical theory, the BIS theory of ADHD is postulated to be due to under-functioning of the septohippocampal system, while LeDoux (2002) places greater emphasis on the amygdala. LeDoux has described the behavioral inhibition system as a network that 'detects and responds to aversive stimuli, those that produce pain, punishment, failure, or loss of reward, or that elicit novelty and uncertainty,' resulting in inhibition of ongoing behavior with increased arousal and vigilance. LeDoux suggests that fear (reaction to a present stimulus) and anxiety (concern about what might happen) can be separated into amygdala-based and hippocampal/prefrontal networks, but differs from Gray in attributing a greater role to the amygdala than hippocampus in threat processing.

Despite the considerable interest aroused by the work of both Quay and Barkley, there have remained features of ADHD, not explained by either theory. Barkley (1997a) reviewed the evidence supporting a deficiency in behavioral inhibition in ADHD. This included behavioral observations of impulsivity, difficulty in conforming to instructions, difficulty in inhibition of prepotent responses, and poor interference control.

According to Barkley (1997a), a new theory of ADHD was needed because current research was 'nearly a-theoretical, at least as regards its basic nature,' with the exception of the
Quay/Gray model and the work of Sergeant, van der Meere and colleagues (Sergeant, 1988, 1995; Sergeant and van der Meere, 1988; van der Meere et al, 1989). Sergeant et al (1999) argued that a poor inhibitory deficit in ADHD was insufficiently specific to conclude that the core of ADHD is an inhibition deficit, because poor inhibitory performance is not specific to ADHD. They argued that energetic factors are critical to the performance deficits of ADHD. According to Barkley (1997a) these researchers successfully employed information processing theory and its associated energetic model (arousal, activation and effort) to isolate central deficits in ADHD, but did not provide a large-scale theory construction, so as to provide a unifying account of the various cognitive deficits associated with ADHD. Barkley also suggested that a deficit in behavioral inhibition in ADHD should be associated with less affective and motivation self-control, but concluded that emotional selfcontrol could also be associated with frustration due to cognitive deficits such as working memory or comorbid learning disorder.

The present theory adds the concept of impaired tonic/ phasic DA relationships in mesolimbic DA systems giving rise to impaired reward mechanisms and impulsivity (BAS); and secondly a deficit in synaptic gating processes in specific ventral striatal (accumbens) synapses resulting in comorbid anxiety. These processes may be interrelated by cortical/subcortical re-entrant circuits as described by Heimer (2003) below.

\section{Comorbidity and ADHD}

Epidemiological studies of ADHD (Graetz et al, 2001) have reported higher scores on the Anxious/Depressed scale of the Achenbach Child Behavior Checklist (CBCL), and on all three Externalizing scales of the CBCL. Some studies suggest that $50-80 \%$ of children with ADHD also meet diagnostic criteria for other disruptive behavior disorders, namely Oppositional Defiant Disorder (ODD) (Waldman and Lilienfeld, 1991) and Conduct Disorder (CD) (Thapar et al, 2001) or for Learning Disorders and Communication Disorders (Tannock, 1998). Waldman et al (2001) showed considerable overlap in the genetic and environmental influences on ADHD, CD, and ODD.

There are also higher rates for internalizing problems such as anxiety (Goodyear and Hynd, 1992). Pliszka (2000) reviewed studies, which examined the comorbidity of ADHD with ODD, CD, and affective, anxiety, and learning disorders. He reported that anxiety and ADHD appeared to be inherited independently and that studies examining stimulant response in children with $\mathrm{ADHD} /$ anxiety have yielded conflicting results.

Pliszka et al (1999) suggested that approximately $25 \%$ of children with ADHD had comorbid anxiety disorders, but indicated that this figure could be affected by changes in diagnostic systems and possible symptom overlap.

Pliszka (1992) reported that ADHD subjects with comorbid anxiety had a significantly poorer response to stimulant medication than those without anxiety, suggesting they might represent children who develop secondary inattentiveness, or perhaps a different type of ADHD. The effects of MPH on working memory and behavior in anxious and nonanxious children with ADHD have been 
investigated (Tannock et al, 1995a). They found that MPH improved working memory in the nonanxious group but not in the comorbidly anxious group. Thus, while there are suggestions that comorbid ADHD/anxiety may represent a distinct subgroup, there are few etiological studies of the comorbidity of childhood anxiety and ADHD, and fewer theoretical explorations.

The MTA study of ADHD (1999) was confined to children with ADHD Combined Type, and showed that in this group, combined medication treatments offered greater benefits than community care for oppositional/aggressive behaviors, internalizing symptoms, peer interactions, and parent-child relations. The present model suggests a biological basis for these findings, in terms of affective regulation provided by more optimal synaptic gating mechanisms resulting from medication effects.

The stop signal task in which the subject is required to stop an ongoing behavior in response to a signal has been shown to be relatively specific to ADHD and to improve with stimulant treatment (Tannock et al, 1995b). The task is considered to activate prefrontal cortex (PFC) and basal ganglia. Impaired prefrontal activation in ADHD has been reported by Silberstein et al (1998).

\section{Architecture of Nucleus Accumbens (NAcc)/Ventral Striatum}

Mogenson et al (1980) described the role of the NAcc in functioning as an interface between limbic and motor systems ('from motivation to action'). Mogenson quoted Graybiel's findings that the NAcc is a key structure in linking motivation and action at the interface of the limbic system with motor mechanisms, receiving direct connections from amygdala, hippocampus, and other limbic forebrain structures, as well as indirect connections via mesolimbic dopaminergic projections from the ventral tegmental area. The NAcc has direct motor connections to the globus pallidus and indirect connections via the substantia nigra and nigrostriatal dopaminergic system. Mesolimbic DA projections to the NAcc in rats were also implicated by abolition of the hyperactivity effect of systemically administered amphetamine by damage to ventral tegmental projections to the NAcc by injections of 6-hydroxydopamine.

According to Dreyer (http://www.unifr.ch/biochem/ DREYER/Brain\%20Areas/striatAcc.htm), the ventral striatum including the NAcc receives input from cortical areas other than the motor and sensory areas of the frontal and parietal lobes. It receives input mainly from areas that do not project to the dorsal striatum notably the temporal (including the hippocampal formation), limbic, and orbitofrontal cortical areas as well as the basolateral amygdala. The ventral striatum in turn projects to the ventral pallidum. Information is then relayed via the dorsomedial and ventral anterior nuclei of the thalamus to the cingulate, orbitofrontal and PFC and to the ventral tegmental area of the brainstem. The ventral circuitry parallels the dorsal circuitry and is considered to be involved with behavioral phenomena, reward and punishment, and in integrating cognitive with emotional responses. Thus, the ventral striatum, including the accumbens is considered critical in integrating cognitive and emotional responses.
Groenewegen et al (1997) demonstrated by labelling and tracing experiments in rats, the topography of a number of circuits involving PFC, ventral striatum, and the mediodorsal thalamic nucleus. This arrangement was considered to be consistent with parallel functionally segregated basal ganglia-thalamocortical circuits, which pass from distinct parts of the (pre)frontal cortex via the striatum, pallidum/ substantia nigra, thalamus, and back to premotor/PFC.

Heimer (2003) has described the ventral striatal (including accumbens), pallidal system and extended amygdala as the major components of the 'new anatomy' of the basal forebrain. He describes three re-entrant circuits - the anterior cingulate, the lateral orbito-frontal, and the medial orbito-frontal, which are closed in that they originate and terminate in the same area of the frontal lobe, but suggests that they can also interact. He suggests that the entire cerebral cortex including the hippocampus and major parts of the amygdala project to the basal ganglia and are involved in the regulation of specific emotional functions and adaptive behaviors ranging from fear-anxiety, and addictive-reward, and appetitive behavior. The ventral striatum integrates various cortical and subcortical inputs to adapt 'motivational' behavior in a similar way to that in which the motor loop through the dorsal parts of the basal ganglia is important for movement control.

French and Totterdell (2002) used in vivo injection of biotinylated dextran amine (BOA) in rats to demonstrate that individual accumbens cells received afferent input from both the ventral subiculum of the hippocampus and the PFC. They postulated that the accumbens (a central component of the ventral striatum) is centrally positioned to integrate signals arising from limbic and cortical areas, and thus modulate motor output related to goal-directed behavior. The investigators believed this monosynaptic convergence to be the first anatomical support for the hypothesis that hippocampal input to the accumbens 'gates' input from the PFC as postulated by O'Donnell and Grace (1995) who showed that accumbens neurons manifest bistable membrane potentials, alternating between a hyperpolarized and a relatively depolarized state, which is more likely to fire action potentials in response to PFC inputs when depolarized. French and Totterdell (2003) examined synaptic input to the projection neurons of the accumbens, the medium-sized density spiny neurons by labelling with biottinylated dextran amine. They demonstrated anatomically that monosynaptic convergence between the ventral subicular region of the hippocampus and the basolateral region of the amygdala occurred at the level of proximal as well as distal dendrites. This anatomical arrangement with the accumbens was similar to that demonstrated above between PFC and the ventral subiculum and accumbens, providing anatomical support for the hypothesis that the PFC, hippocampus, and amygdala are capable of monosynaptic gating at the level of the medial shell of the accumbens. They postulated that a reciprocal arrangement allowing an opposing gating action of limbic input upon another function at accumbens level, might allow either excitatory or inhibitory postsynaptic responses. This mechanism was also considered to allow integration and association of information from both hippocampus (spatial information) and amygdala (association between primary reinforcer and conditioned stimuli). French et al 
(2003) examined the synaptic input from the basolateral amygdala to the spiny pyramidal projection neurons of the hippocampal subiculum. Electron microscopy demonstrated anatomically that the basolateral amygdala preferentially innervates spiny subiculum neurons, providing a possible synaptic mechanism underlying memory enhancement associated with emotions.

\section{Synaptic Gating Theory}

Spinelli and Pribram (1966) demonstrated that processes, which delay recovery from afferent inputs, effectively discouple or inhibit input systems, while any parameter that enhances recovery effectively links input systems. Thus recovery or inhibition of synaptic neurotransmission is likely to be a central factor in the control of affective states.

Physiologically based models have been amplified by pharmacological techniques, which allow investigation of neurotransmitter functions in cortico-limbic systems. Grace $(1995 ; 2000 \mathrm{a} b)$ suggested that when DA is released into the striatal synaptic cleft in response to action potentials, it is rapidly removed from the synapse by a highly efficient reuptake system. On the other hand, tonic DA level is considered to be mediated by stimulation of presynaptic heteroreceptors on DA terminals by corticostriatal glutamergic projections. Tonic DA exerts a suppressive influence on subcortical DA systems and is regulated, in part by frontal and cortical afferents to the accumbens (Figure 1).

The NAcc receives input from a number of limbic-related cortical structures, including the PFC, hippocampus, and amygdala (Grace, 2001). In particular, the hippocampus and amygdala strongly influence the ability of the PFC to activate accumbens cell firing. As a result these systems alternately modulate the flow of information from the PFC through the NAcc, where it can ultimately influence thalamocortical function via projections through the ventral a

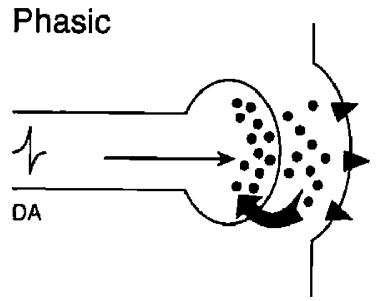

c Tonic Modulates Phasic

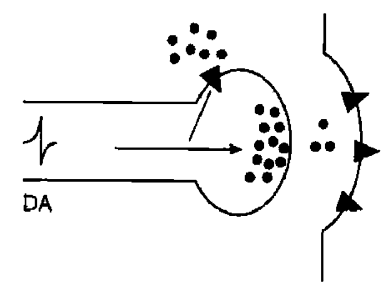

b

Tonic
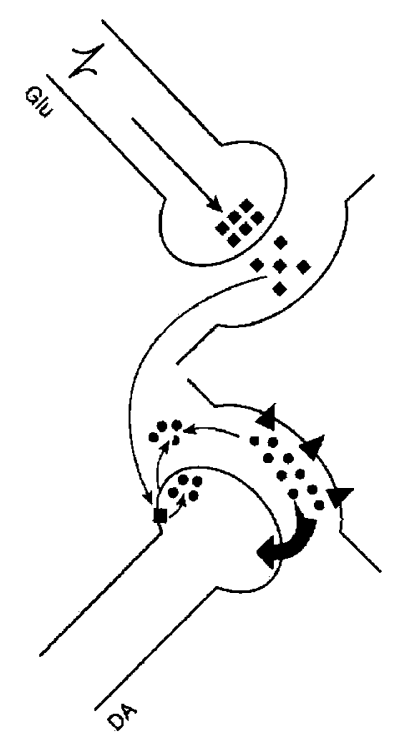

Figure I Tonic/Phasic Model of DA System Function. pallidum. According to Grace (2001) the NAcc is the striatal region in which the limbic system has overlapping inputs. As described above, accumbens neurons exist in a bistable state, with their membrane potential alternating between a hyperpolarized nonfiring state and a depolarized plateau lasting several hundred milliseconds, during which spike activity is generated.

Grace (2001) also suggests that the amygdala gates events based on their affective valence. 'The amygdala also influences the accumbens by facilitating prefrontal stimuli but only within a very narrow single-event related timeinterval.' Thus, this bistable accumbens state allows the operation of a synaptic gating mechanism between prefrontal and limbic influences on behavior. 'Studies have suggested that the subiculum of the hippocampus is involved in a type of context-dependent gating influence over the ability of the PFC to control thalamocortical information processing, enabling the organism to stay ontask and focused. This mesocortical DA system provides a potent direct influence overactivity within the PFC. Among the many functions of the PFC is the generation of a set of potential responses that the subject can utilize to react to a stimulus. Thus, while the hippocampus generates long duration activity in the accumbens neurons, keeping the subject focussed on the current task, the amygdala provides a more, brief event-related gating of prefrontal throughput in the accumbens.'

Goto and O'Donnell (2002) have reported timingdependent limbic-motor synaptic integration in the NAcc. They used in vivo intracellular recordings to record excitatory postsynaptic potentials (EPSPs) in the lateral shell and medial core region of the NAcc, after stimulation from the ventral subuliculum, prelimbic cortex, posterior basolateral amygdala, or paraventricular nucleus of the thalamus. They showed that coincident PFC and limbic inputs reduce EPSP variability, suggesting that correlated activity between the PFC and limbic structures results in stable activity in the accumbens, whereas asynchronous inputs depend on the order of arrival: limbic inputs enhance subsequent PFC inputs, and PFC inputs dampen subsequent limbic-evoked EPSPs, possibly via feed-forward inhibition by interneurons or axon collaterals of projection neurons. While the pharmacological mechanism is not well understood, the investigators suggest that accumbens neurons fluctuate between UP states (membrane potential staying UP for at least $100 \mathrm{~ms}$ ), which might involve persistent activation of ionic currents that facilitate both simultaneous and subsequent PFC inputs, while the DOWN state, a negative resting potential requires strong barrage of limbic inputs in order to depolarize accumbens neurons. Correlated limbiccortical activity is important for cognitive functions, and may be a means of appropriate behavior selection. In the current context the demonstration of differing effects of coincident and asynchronous PFC and limbic activity suggests a possible gating mechanism, whereby PFC activity can gate limbic input depending on the state of the neurons and the timing of inputs.

According to Grace (2001) the amygdala is involved in emotional or affective properties of stimuli, enabling the subject to respond to events that are emotionally charged, and therefore of immediate survival value. In pathological states, the amygdala input may be overdriven to the extent 
that the maintenance of focus is overly disrupted by minor events. As described by Arnsten (2000) high levels of DA and norepinephrine may have additive effects on information processing in $\mathrm{PFC}$, reducing signals and increasing noise. Arnsten points out that although PFC functions are often essential for successful organization of higher-order behavior, there may be some conditions, when it may be adaptive to 'shut down' these complex, reflective operations and to allow more automatic or habitual responses, dependent on posterior cortical or subcortical structures to control behavior.

Schultz (2000) and co-workers (Suri and Schultz, 2001; Mirenowicz and Schultz, 1994) have used single neuron recording techniques in monkeys to investigate the conditions in which DA neurons respond to rewarding or potentially rewarding stimuli. They have shown that dopaminergic neurons in the VTA (A8, A9, A10), which project to the NAcc and frontal cortex show short phasic activation after reward presentations, and visual or auditory stimuli that predict reward. By contrast only a few dopamine neurons show phasic activations to punishers (conditioned aversive visual or auditory stimuli).

Monoaminergic disruptions of amygdala, PFC, and accumbens equilibrium are likely to be involved in a number of psychopathologies. Sagvolden et al (2004) have postulated that the behavior and symptoms of ADHD derive from altered dopaminergic function with a consequent failure to modulate nondopaminergic (predominantly glutamate and GABA) signal transmission. They postulate that a hypo-functioning mesolimbic dopamine branch produces altered reinforcement of behavior and deficient extinction of previously reinforced behavior, giving rise to delay aversion, impulsivity, and failure to 'inhibit' responses or disinhibition. A hypo-functioning mesocortical branch is postulated to cause attentional deficits, while a hypofunctioning nigrostriatal branch gives rise to impaired motor functions.

Sagvolden et al (2004) have argued that the main component of altered reinforcement processes in ADHD children, is a steeper delay-of-reinforcement gradient, or time interval between response and effective reinforcer, resulting in less effective reinforcement and also less effective extinction of previously established, but no longer reinforced responses. This explains why ADHD childrens' behavior is temporarily less impulsive in response to potent and frequent reinforcers. When a delay gradient quickly drops to zero responses must be very close to a reinforcer to be captured by it and only a single response will be captured. The implication of a steep reward gradient in ADHD implies a reduced capacity for reward (operant conditioning), hence the indifference to social acceptability often manifested as oppositional or conduct problems.

Sagvolden et al (2004) point out that children's behavior is gradually brought under discriminative control by the establishment of verbally governed behavior (responsive to verbal reward).

Schultz et al (1993) studied the responses of dopamine neurons during the steps of learning a behavioral task. They showed that in monkeys trained to perform a spatial delayed response task, via two intermediate tasks, dopamine neurons showed responses during, but much less, after learning each task. These responses were most pronounced in area A10 as compared to areas A8 and A9. Dopamine neurons also showed phasic responses to instructional and conditioned incentive stimuli predicting reward, but did not exhibit activity during the delay between instructions and triggers. The investigators concluded that while dopamine neurons respond phasically to alerting stimuli necessary for task learning, they do not encode representational processes such as working memory, rather they respond phasically to basic attentional and motivational stimuli involved in learning. Goldman-Rakic (1990) has contrasted the type of behavioral regulation subserved by PFC with stimulusresponse associations. The former 'delayed response' abilities depend on the maintenance of representational memory, while the latter depend on associative conditioning to innate prepotent responses, usually at subcortical level. She describes a functional dissociation between working and associative memory.

According to Floresco et al (2003), the mesolimbic dopamine system plays a central role in reward and goaldirected behavior, and has been implicated in multiple psychiatric disorders. Understanding the mechanism by which dopamine participates in these activities requires comprehension of the dynamics of dopamine release. They report dissociable regulation of dopamine neuron discharge by two separate afferent systems in rats; inhibition of pallidal afferents selectively increases the population activity of dopamine neurons, whereas activation of pedunculo-pontine inputs increases burst firing.

According to Floresco et al (2003) synaptic or phasic levels of dopamine are mediated by bursting events at the level of the cell body, restricted by high affinity and rapid uptake systems, and associated with reward-conditioned prediction. On the other hand, extrasynaptic or tonic dopamine levels are modulated by presynaptic limbic and cortical glutamergic inputs. Alterations in tonic levels of dopamine efflux occur on a much slower time-scale and allow a wide variety of motor, cognitive, and motivational functions. The results provide insight into multiple regulatory systems that modulate dopamine system function; burst firing inducing massive synaptic dopamine release, rapidly removed by reuptake before escaping the synaptic cleft, whereas increased population activity modulates tonic extrasynaptic dopamine levels that are less influenced by reuptake, and presumably affect long-term disposition. In ADHD children, impairment of tonic/phasic relationships may influence reinforcement gradients described by Sagvolden et al (2004), as a result of lowered availability of tonic DA levels in the mesolimbic and mesocortical systems, resulting in the stimulus-bound, impulsive 'fearless' behavior of ADHD children. On the other hand, impaired synaptic gating by PFC at the accumbens level allows greater access to conditioned amygdala reactions and the anxiety (or aggression) described in some ADHD children.

Davis and Whalen (2001) have comprehensively reviewed animal and human literature concerning the role of the amygdala in fear conditioning. They found that the basolateral amygdala is involved in negative and positive affect, as well as spatial and motor learning. They describe the 'extended amygdala' as target areas that subserve more specialized functions. These target areas are critical for the specific symptoms of fear, as well as the experience of fear. 
They believe the complex behavioral pattern seen during a state of 'conditioned fear' has already been hard-wired during evolution, and that it is only necessary for a stimulus to activate the amygdala following aversive conditioning.

\section{DISCUSSION}

The paradoxical apparently 'fearless' behavior of ADHD children, yet the presence of anxiety in some ADHD children may be explained by separate reward and fear conditioning processes, as postulated by Gray (1975). The former appears to be related to impaired operant conditioning processes secondary to impairment of mesolimbic tonic/phasic DA relationships, while fear conditioning is based on inadequate PFC and hippocampal synaptic gating of classically conditioned noradrenergic and serotonergic responses in the amygdala.

The demonstration by French and Totterdell $(2002,2003)$ of monosynaptic convergence between the ventral subicular region of the hippocampus, the basolateral region of the amygdala and PFC on accumbens dendrites provides anatomical support for the hypothesis that the PFC and hippocampus are capable of monosynaptic gating of amygdala input at the level of the medial shell of the accumbens.

Floresco et al (2001) have shown that the ventral subiculum is involved in regulating the basal firing characteristics as well as the overall activity level of VTA DA neurons in the normal rat. If disrupted early in development, tonic control of VTA DA neuron activity is affected, so that phasic activation of DA neuron firing has an abnormally large impact on impulse dependent DA release in limbic structures, as postulated by O'Donnell and Grace (1995), giving rise to heightened limbic DA responsivity. This would be predicted to give rise to an increase in immediate indiscriminate reward seeking behavior (fearlessness) and overactivity, as described by Sagvolden and co-workers.

On the other hand, the reduction of PFC and hippocampal synaptic DA gating at the accumbens level allows increased amygdala-based fear or anxiety to be manifest in some (possibly genetically predisposed) ADHD children. Thus comorbidity in ADHD children is complex and related to the tonic/phasic DA relationships in the VTA giving rise to 'fearless' stimulus-bound externalizing behavior as a result of impaired reinforcement gradients in some ADHD children, while abnormal PFC and hippocampal gating of amygdala-based anxiety is observed in other ADHD children. The present distinctions provide a basis for possible pharmacological approaches to the treatment of comorbidity in ADHD. For example, stimulant medications, which increase tonic dopamine levels are likely to be useful for impulsivity and fearlessness, whereas axiolytic medications such as specific serotonin re-uptake inhibitors (SSRIs) or combined nor-adrenergic/dopaminergic antidepressants may be useful for comorbid anxiety. The use of dopamine partial agonists, which are postulated to have differential effects in mesolimbic and mesocortical pathways, may also be of interest (Lieberman, 2004).

While the above discussion relates to comorbid fearlessness and impulsivity $v s$ anxiety, it does not address the issue of comorbid aggression. Blair (2001) has described two forms of aggression, reactive aggression elicited in response to frustration/threat and goal-directed instrumental aggression. He suggests that impairment in the capacity to form associations between emotional unconditioned distress cues and conditioned stimuli is related to the instrumental aggression shown by persons with 'developmental psychopathy.' Kilcross et al (1997) have described different types of fear-conditioned behavior mediated by separate nuclei within amygdala, supporting different forms of fear-related behavior. They describe limbic cortico-ventral striatopallidal circuitry, which provides an interface between the processing of emotionally salient stimuli and intentional action, while areas of the orbital PFC that have significant reciprocal connections with the basolateral amygdala are implicated in the assignment of affective or 'somatic' markers influencing behavior. While these influences are complex they also appear to be related to dual cortical/ instrumental and amygdala conditioned influences on aggression, either or both of which could be impaired in a re-entrant circuit influencing aggression in ADHD children. The concept of ventral re-entrant circuits elaborated by Heimer (2003) above allows a useful integration of tonic/ phasic relationships at mesolimbic and mesocortical levels with gating processes at the amygdala. Impairments of these circuits may also give rise to a number of other neuropsychiatric conditions such as schizophrenia or autism depending on genetic and developmental influences.

\section{ACKNOWLEDGEMENTS}

I thank Professor Richard Todd, Dr Mina Dulcan, and Professor Anthony Grace for their helpful comments on the manuscript, and Dr John Merson and Professor Gordon Parker for their support of the thesis, which gave rise to the present model.

\section{REFERENCES}

Arnsten AFT (2000). Stress impairs prefrontal cortical function in rats and monkeys: role of dopamine $\mathrm{d} 1$ and norepinephrine $\alpha-1$ receptor mechanisms. Prog Brain Res 126: 183-192.

Barkley RA (1997a). ADHD and The Nature of Self-Control. The Guilford Press: New York, NY.

Barkley RA (1997b). Behavioral inhibition, sustained attention, and executive functions: constructing a unifying theory of ADHD. Psychol Bull 121: 65-94.

Biederman J, Faraone SV, Keenan K, Krifcher B, Moore C, SprichBuckminster S et al (1992). Further evidence for family-genetic risk factors in Attention Deficit Hyperactivity Disorder. Arch Gen Psychiatry 49: 728-738.

Biederman J, Rosenbaum JF, Bolduc-Murphy EA, Faraone SV, Chaloff J, Hirshfield DR et al (1993). A 3-year follow-up of children with and without behavioural inhibition. J Am Acad Child Adolescent Psychiatry 32: 814-821.

Blair RJR (2001). Neurocognitive models of aggression, the antisocial personality disorders, and psychopathy. Adv Neuropsychiatry 71: 727-731.

Davis M, Whalen PJ (2001). The amygdala: vigilance and emotion. Mol Psychiatry 6: 13-34.

Floresco SB, Todd CL, Grace AA (2001). Glutamatergic afferents from the hippocampus to the nucleus accumbens regulate 
activity of ventral tegmental area dopamine neurons. J Neurosci 21: 4915-4922.

Floresco SB, West AR, Ash B, Moore H, Grace AA (2003). Afferent modulation of dopamine neuron firing differentially regulates tonic and phasic dopamine transmission. Nat Neurosci 6: 968-973.

French ST, Totterdell S (2002). Hippocampal and prefrontal cortical inputs monosyaptically converge with individual projection neurons of the nucleus accumbens. J Comp Neurol 446: 151-165.

French SJ, Totterdell S (2003). Individual nucleus accumbensprojection neurons receive both basolateral amygdala and ventral subicular afferents in rats. Neuroscience 119: 19-31.

French SJ, Hailstone JC, Totterdell S (2003). Basolateral amygdala efferents to the ventral subiculum preferentially innervate pyramidal cell dendritic spines. Brain Res 981: 160-167.

Goldman-Rakic PS (1990). Cortical localization of working memory. In: McGough JL (ed). Brain Organisation and Memory. Oxford University Press: New York. pp 285-298.

Goodyear P, Hynd C (1992). Attention-Deficit Disorder with $(\mathrm{ADD} / \mathrm{H})$ and without $(\mathrm{ADD} / \mathrm{WO})$ hyperactivity: behavioural and neuropsychological differentiation. J Clin Child Psychol 21: 243-253.

Goto Y, O'Donnell P (2001). Synchronous activity in the hippocampus and nucleus accumbens in vivo. J Neurosci 21: RC131.

Goto Y, O'Donnell P (2002). Timing-dependent limbic-motor synaptic integration in the nucleus accumbens. PNAS 99: 13189-13193.

Grace AA (1995). The tonic/phasic model of dopamine system regulation; its relevance for understanding how stimulant abuse can alter basal ganglia function. Drug Alcohol Depend 37: 111-729.

Grace AA (2000a). The tonic/phasic model of dopamine system regulation and its implications for understanding psychostimulant craving. Addiction 95(Suppl 2): S119-S128.

Grace AA (2000b). Gating of information flow within the limbic system and the pathophysiology of schizophrenia. Brain Res Rev 31: $330-341$.

Grace AA (2001). Psychostimulant actions on dopamine and limbic system function: relevance to the pathophysiology and treatment of ADHD. In: Solanto MV, Arnsten AFT, Castellanos FX (eds). Stimulant Drugs and ADH: Basic and Clinical Neuroscience. Oxford University Press: Oxford and New York. pp 134-157.

Graetz BW, Sawyer M, Hazell PL et al (2001). Validity of DSM-IV ADHD subtypes in a nationally representative sample of Australian children and adolescents. J Am Acad Child Adolescent Psychiatry 40: 1410-1417.

Gray JA (1975). Elements of a Two-Process Theory of Learning. Academic Press: London.

Gray JA. (1987). The Psychology of Fear and Stress. Cambridge University Press: New York and Cambridge.

Groenewegen HJ, Wright CI, Ulings HB (1997). The anatomical relationships of the prefrontal cortex with limbic structures and the basal ganglia. J Psychopharmacol 11: 99-106.

Heimer L (2003). A new anatomical framework for neuropsychiatric disorders and drug abuse. Am J Psychiatry 160: 1726-1739.

Hudziac, Todd RD (1993). Familial subtyping of attention deficit hyperactivity disorder. Curr Opin Psychiatry 6: 489-493.

Kilcross S, Robbins TW, Everitt BJ (1997). Different types of fear-conditioned behaviour mediated by separate nuclei within amygdala. Nature 388: $377-380$.

LeDoux J (2002). Synaptic Self. Viking: New York.

Lieberman JA (2004). Dopamine partial agonists: a new class of antipsychotic. CNS Drugs 18: 251-267.

Mirenowicz J, Schultz W (1994). Importance of unpredictability for reward responses in primate dopamine neurons. J Neurophysiol 72: $1024-1027$.
Mogenson GJ, Jones DL, Yim CY (1980). From motivation to action: functional interface between the limbic system and the motor system. Prog Neurobiol 14: 69-92.

O’Donnell P, Grace AA (1995). Synaptic interactions among excitatory afferents to nucleus accumbens neurons: hippocampal gating of prefrontal cortical input. J Neurosci 15: 3622-3639.

Oosterlaan J (2001). Behavioural inhibition and the development of childhood anxiety disorders. In: Silverman WK, Treffers PDA (eds). Behavioural Inhibition and the Development of Childhood Anxiety Disorders: Research, Assessment and Intervention. Cambridge Child and Adolescent Psychiatry: Cambridge. pp 45-71.

Oosterlaan J, Sergeant JA (1996). Inhibition in ADHD, aggressive, and anxious children: a biologically based model of child psychopathology. J Abnormal Child Psychol 24: 19-36.

Pliszka SR (1992). Comorbidity of attention-deficit hyperactivity disorder and overanxious disorder. J Am Acad Child Adolescent Psychiatry 31: 197-203.

Pliszka SR (2000). Patterns of comorbidity with attention-deficit/ hyperactivity disorder. Child Adolescent Psychiatric Clin N Am 9: 525-540.

Pliszka SR, Carlson CL, Swanson JM (1999). ADHD with Comorbid Disorders: Clinical Assessment and Management. Guilford Press: New York.

Quay HC (1988a). Attention deficit disorder and the behavioral inhibition system: the relevance of the neuropsychological theory of Jeffrey A Gray. In: Bloomingdale LM, Sergeant J (eds). Attention Deficit Disorder: Criteria, Cognition, Intervention. Pergamon Press: Oxford, England. pp 117-125.

Quay HC (1988b). The behavioural reward and inhibition systems in childhood behavior disorders. In: Bloomingdale LM (ed). Attention Deficit Disorder. Vol 3: New Research in Attention, Treatment and Psychopharmacology. Pergamon Press: Oxford, England. pp 176-186.

Quay HC (1993). The psychobiology of undersocialized aggressive conduct disorder: a theoretical perspective. Dev Psychopathol 5: $165-180$.

Quay HC (1997). Inhibition and attention deficit hyperactivity disorder. J Abnormal Child Psychol 25: 7-13.

Sagvolden T, Johansen EB, Aase H, Russell VA (2004). A dynamic developmental theory of Attention-Deficit/Hyperactivity Disorder (ADHD) predominantly hyperactive/impulsive and combined subtypes. Beh Brain Sci, in press.

Schultz W (2000). Multiple reward signals in the brain. Nat Rev Neurosci 1: 199-204.

Schultz W, Apicellar P, Llungberg T (1993). Responses of monkey dopamine neurons to reward and conditioned stimuli during successive steps of learning a delayed response task. J Neurosci 13: $900-913$.

Seeman P, Madras BK (1999). Anti-hyperactivity medication: methylphenidate and amphetamine. Mol Psychiatry 3: 386-396.

Sergeant JA (1988). From DSM-III attentional deficit disorder to functional defects. In: Bloomingdale L, Sergeant J (eds). Attention Deficit Disorder: Criteria, Cognition and Intervention. New York: Pergamon Press. pp 183-198.

Sergeant J (1995). Hyperkinetic disorder revisited. In: Sergeant JA (ed). Eunethydis: European Approaches to Hyperkinetic Disorder. JA Sergeant: Amsterdam. pp 7-17.

Sergeant JA, van der Meere JJ (1988). What happens when the hyperactive child commits an error? Psychiatry Res 24: 157-164. Sergeant JA, Oosterlaan J, van der Meere J (1999). Information processing and energetic factors in attention-deficit/hyperactivity disorder. In: Quay HC, Hogan AE (eds). Handbook of Disruptive Behavior Disorders. Kluwer Academic/Plenum Publishers: New York. pp 75-104.

Silberstein RB, Farrow M, Levy F et al (1998). Functional brain electrical activity mapping in boys with Attention-Deficit/ Hyperactivity Disorder. Arch Gen Psychiatry 55: 1105-1111. 
Spinelli DN, Pribram KH (1966). Changes in visual recovery functions produced by temporal lobe stimulation in monkeys. Electroencephalogr Clin Neurophysiol 20: 44-49.

Suri RE, Schultz W (2001). Temporal difference model reproduces anticipatory neural activity. Neur Comput 13: 841-862.

Tannock R (1998). Advances in cognitive, neurobiological and genetic research. J Child Psychol Psychiat 39: 65-99.

Tannock R, Ickowicz A, Schachar R (1995a). Differential effects of methylphenidate on working memory in ADHD children with and without comorbid anxiety. J Am Acad Child Adolescent Psychiatry 34: 886-896.

Tannock R, Schachar R, Logan G (1995b). Methylphenidate and cognitive flexibility: dissociated dose effects in hyperactive children. J Abnormal Child Psychol 23: 235-266.

Thapar A, Harrington R, McGuffin P (2001). Examining the comorbidity of ADHD-related behaviours and conduct problems using a twin study design. $B r J$ Psychiatry 179: 224-229.

The MTA Cooperative Group (1999). A 14-month randomised clinical trial of treatment strategies for Attention-Deficit/ Hyperactivity Disorder. Arch Gen Psychiatry 56: 1073-1086.

van der Meere J, van Baal M, Sergeant J (1989). The additive factor method; a differential diagnostic tool in hyperactivity and learning disability. J Abnormal Child Psychol 17: 409-422.

Waldman ID, Lilienfeld SO (1991). Diagnostic efficiency of symptoms for Oppositional Defiant Disorder and Attention-Deficit Hyperactivity Disorder. J Consulting Clin Psychol 39: 732-738.

Waldman ID, Rhee SH, Levy F, Hay DA (2001). Causes of the overlap among symptoms of ADHD, Oppositional Defiant Disorder, and Conduct Disorder. In: Levy F, Hay D (eds). Attention, Genes and ADHD. Brunner-Routledge: Hove, East Sussex. pp 115-138. 